\title{
Spermidine promotes stress resistance in Drosophila melanogaster through autophagy-dependent and -independent pathways
}

\author{
N Minois ${ }^{\star, 1}$, D Carmona-Gutierrez ${ }^{\star, 2}$, MA Bauer ${ }^{2}$, P Rockenfeller ${ }^{2}$, T Eisenberg ${ }^{2}$, S Brandhorst ${ }^{3,4}$, SJ Sigrist ${ }^{5,6}$, G Kroemer $^{7,8,9,10}$ \\ and $\mathrm{F} \mathrm{Madeo}^{2}$
}

The naturally occurring polyamine spermidine (Spd) has recently been shown to promote longevity across species in an autophagy-dependent manner. Here, we demonstrate that Spd improves both survival and locomotor activity of the fruit fly Drosophila melanogaster upon exposure to the superoxide generator and neurotoxic agent paraquat. Although survival to a high paraquat concentration $(20 \mathrm{mM})$ was specifically increased in female flies only, locomotor activity and survival could be rescued in both male and female animals when exposed to lower paraquat levels $(5 \mathrm{mM})$. These effects are dependent on the autophagic machinery, as Spd failed to confer resistance to paraquat-induced toxicity and locomotor impairment in flies deleted for the essential autophagic regulator ATG7 (autophagy-related gene 7). Spd treatment did also protect against mild doses of another oxidative stressor, hydrogen peroxide, but in this case in an autophagy-independent manner. Altogether, this study establishes that the protective effects of Spd can be exerted through different pathways that depending on the oxidative stress scenario do or do not involve autophagy.

Cell Death and Disease (2012) 3, e401; doi:10.1038/cddis.2012.139; published online 11 October 2012

Subject Category: Experimental Medicine

The population's proportion of older people is steadily increasing in many countries and with it the incidence of experiencing the process of ageing. More people will live longer ${ }^{1}$ but will also have an elevated risk of suffering ageassociated disabilities and diseases. Thus, being able to postpone and/or lessen the deleterious effects of ageing represents an acute challenge for modern society and would bring high societal and economical advantages.

Our understanding of ageing has increased at an unprecedented pace during the last 30 years and we have now realised that ageing is a plastic process that can be modulated. Indeed, longevity is partly under genetic control and mutations in single genes have been shown to increase life span and, interestingly, also stress resistance in model organisms. ${ }^{2}$ Alternatively, ageing can be modulated by external, non-genetic interventions. One of them is dietary restriction, where a decrease in the amount of food intake delays ageing, disease onset and mortality in a wide range of organisms, including non-human primates. ${ }^{3}$ Furthermore, several pharmacological interventions have been recently reported to be beneficial for ageing, diseases and life span extension, even though the obtained results do not offer a clear picture. For instance, resveratrol, a naturally occurring phenol found, for example, in the skin of red grapes, increased the life span of mice kept on a high-fat diet. ${ }^{4}$ However, the doses used to achieve life span extension were very high, raising the question of this compound's bioavailability. In addition, resveratrol has not been shown to exert any beneficial effect in healthy organisms. ${ }^{5}$ The immunosuppressant drug rapamycin has also been demonstrated to increase the life span of rodents ${ }^{5,6}$ but only shows inconclusive effects in the fruit fly Drosophila melanogaster. ${ }^{7,8}$ Notably, a serious drawback of rapamycin is its immunosuppressant properties, which could hinder its potential use on a wide basis in healthy organisms. Altogether, these examples clearly show that further research is needed to broaden our knowledge on the effects and mechanisms governing the activity of already identified molecules and to find new ones.

Spermidine ( $\mathrm{Spd}$ ) is a natural polyamine involved in an array of crucial molecular processes such as DNA stability,

\footnotetext{
${ }^{1}$ School of Biology, Biomedical Sciences Research Complex, University of St. Andrews, St. Andrews, UK; ${ }^{2}$ Institute for Molecular Biosciences, University of Graz, Graz, Austria; ${ }^{3}$ Andrus Gerontology Center, Department of Biological Sciences and Norris Cancer Center, University of Southern California, Los Angeles, CA, USA; ${ }^{4}$ Centre for Medical Biotechnology, Faculty of Biology, University Duisburg-Essen, Essen, Germany; ${ }^{5}$ Department of Genetics, Institute for Biology, Free University Berlin, Berlin, Germany; ${ }^{6}$ Neurocure Cluster of Excellence, Charité Berlin, Berlin, Germany; ${ }^{7}$ INSERM, U848, Institute Gustave Roussy, University Paris XI, Villejuif, France; ${ }^{8}$ Metabolomics Platform, Institut Gustave Roussy, Villejuif, France; ${ }^{9}$ Centre de Recherche des Cordeliers, Université Paris Descartes, Paris 5, Paris, France and ${ }^{10}$ Pôle de Biologie, Hôpital Européen Georges Pompidou, AP-HP, Paris, France

${ }^{*}$ Corresponding author: N Minois, School of Biology, Biomedical Sciences Research Complex, University of St. Andrews, St. Andrews, Fife KY16 9ST, UK. Tel: + 44133446 3586; Fax: + 44709284 9868; E-mail: nm61@ st-andrews.ac.uk

or D Carmona-Gutierrez, Institute for Molecular Biosciences, University of Graz, Humboldtstrasse 50/EG, Graz 8010, Austria. Tel: +43 3163801499 ; Fax: + 43316380 9898; E-mail: carmonag@uni-graz.at

Keywords: Drosophila melanogaster, spermidine; paraquat; oxidative stress; activity; starvation

Abbreviations: ATG7, autophagy-related gene 7; Spd, spermidine
}

Received 27.3.12; revised 31.7.12; accepted 03.9.12; Edited by M Piacentini 
transcription, translation, apoptosis, cell proliferation, differentiation and survival. Intriguingly, its intracellular level decreases with age. ${ }^{9,10}$ We have recently shown that addition of Spd to the food medium increases the life span of yeast, worms, flies and the survival of human immune cells in culture. ${ }^{11}$ Spd also reduced age-related oxidative damage in mice and increased resistance to hydrogen peroxide $\left(\mathrm{H}_{2} \mathrm{O}_{2}\right)$ and heat in yeast. We further showed that Spd induced intracellular self-digestion (autophagy) to exert its life span extension effect, which could be abrogated by genetic inactivation of autophagy genes in mutant yeast, worms and flies. The regulatory mechanism underlying this effect might be of epigenetic origin. We observed that in yeast, Spd inhibits histone acetyltransferases activity and leads to a global hypoacetylation of histone $\mathrm{H} 3$ at all acetylation sites located at the amino terminus of the histone. Consistent with the antiageing potential of Spd, its intracellular reduction decreased the life span of mice. ${ }^{12}$ Accordingly, in a further report external feeding with polyamines increased life span and reduced ageassociated pathology in a short-lived mouse model. ${ }^{13}$ However, the latter results need to be confirmed as the study was stopped when a significant amount of mice were still alive (after 88 weeks of age). Taken together, these results suggest that Spd could be a powerful tool against the deleterious consequences of ageing. ${ }^{14}$

Anti-ageing properties are often correlated with high stress resistance. ${ }^{15,16}$ In the present study, we address the effects of Spd on two ageing-relevant stresses, oxidative stress and starvation, in the fruit fly $D$. melanogaster. To this end, we first challenged flies with the herbicide paraquat, which is a neurotoxic agent widely employed to generate oxidative stress through the reactive oxygen species superoxide. We show that upon paraquat exposure, treatment with Spd improves survival in female flies exposed to high paraquat levels $(20 \mathrm{mM})$. In addition, it confers longer retention of locomotor activity and survival in both male and female animals when challenged with a lower paraquat concentration $(5 \mathrm{mM})$. We also demonstrate that these effects are exerted in an autophagy-dependent manner. Spd also increases resistance to a different oxidative stressor, $\mathrm{H}_{2} \mathrm{O}_{2}$, when applied at mild doses (1\%). In contrast to paraquat, however, this resistance is not dependent upon functional autophagy, hinting to differential pathways governing Spd-mediated resistance to different oxidative stressors. Finally, we show that Spd fails to rescue toxicity induced by more severe $\mathrm{H}_{2} \mathrm{O}_{2}$ levels $(2 \%)$ or starvation. These results suggest that Spd protects against toxicity resulting from detrimental pathways that involve selective oxidative stress. Furthermore, this protection can be exerted via different mechanisms (that do or do not involve autophagy) depending on the oxidative stress scenario.

\section{Results}

Spd improves survival during $20 \mathrm{mM}$ paraquat stress in female Drosophila. Paraquat is a superoxide generator that has been used, for instance, as a neurotoxic agent to model age-related neurodegenerative diseases in the fruit fly D. melanogaster. ${ }^{17}$ To test the oxidative stress response upon Spd treatment, flies were exposed to $20 \mathrm{mM}$ paraquat and either fed Spd or left untreated. As expected, paraquat strongly compromised fly survival, killing $50 \%$ of the untreated animals (males and females) in average after approximately $55 \mathrm{~h}$ of exposure (Figures $1 \mathrm{a}$ and b). When treated with Spd, female flies challenged with paraquat displayed a significantly better mean and maximum survival, reaching the overall largest improvement at a Spd concentration of $0.1 \mathrm{mM}$ (Figures 1a and c). In males on the other hand, the additional treatment with Spd did not influence paraquat-induced toxicity over the tested concentration range (Figures $1 \mathrm{~b}$ and $\mathrm{d}$ ). For variance between replicates, refer to the Supplementary Material (Supplementary Figures S1A, B). These data suggest that Spd confers sex-specific paraquat resistance, specifically favoring survival in female flies.

Spd increases survival and climbing activity during $5 \mathrm{mM}$ paraquat exposure in both sexes. In addition to detrimental oxidative stress, paraquat also induces locomotor impairment. Thus, we next decided to determine whether Spd might influence locomotor activity upon paraquat exposure. For this purpose, we monitored the ability of flies to climb the vertical wall of the vial in which they were kept, until no fly could perform the task. To decelerate the incidence of death and follow their locomotor activity for a longer period of time, flies were exposed to a lower concentration of paraquat compared to the above survival experiments ( $5 \mathrm{mM}$ instead of $20 \mathrm{mM})$. Indeed, this concentration allowed prolonged overall survival compared to $20 \mathrm{mM}$ (compare Figures $1 \mathrm{a}$ and $\mathrm{b}$ with Figures $1 \mathrm{e}$ and $\mathrm{f}$ ). In addition, treatment with $0.1 \mathrm{mM}$ Spd - the concentration having shown the biggest survival increase to $20 \mathrm{mM}$ paraquat - improved survival to paraquat, and this time in both sexes (Figures 1e-g, Supplementary Figures S1C, D). Similarly, the climbing activities of both female and male flies fed with $0.1 \mathrm{mM}$ Spd was improved compared with the untreated control (Figures $1 \mathrm{~h}$ and i, Supplementary Figures $\mathrm{S} 1 \mathrm{E}-\mathrm{J})$. At $96 \mathrm{~h}$ after the beginning of paraquat exposure, for instance, Spd-treated females displayed about 30\% higher activity than control flies (Figure 1h). In male flies, similar rates were obtained (Figure 1i). Altogether, these data demonstrate that Spd can lessen death, as well as locomotor impairment in both male and female flies, upon exposure to low but toxic paraquat doses $(5 \mathrm{mM})$.

Spd-improved survival and climbing activity are dependent on autophagy. Spd-mediated survival improvement during ageing is at least partly associated with autophagy, ${ }^{11}$ a self-digestion mechanism that has been connected to longevity in various organisms. ${ }^{18}$ To test if this process is involved in Spd-induced paraquat resistance, we performed survival and climbing activity experiments in loss-of-function mutants for atg7 (autophagy-related gene 7) $\left(\operatorname{atg} 7^{-1}\right.$ ), a gene essential for autophagy. In contrast to the results obtained in wild-type flies, in autophagy mutants Spd treatment did not improve the survival decrease caused by paraquat exposure, neither in females with $20 \mathrm{mM}$ nor in both sexes with $5 \mathrm{mM}$ paraquat (Figures 2a-g, Supplementary Figures S2A-D). In fact, in the autophagy-depleted background, Spd treatment even seemed to decrease female survival as compared with 


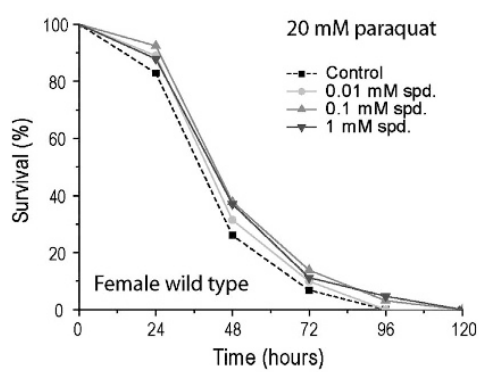

b

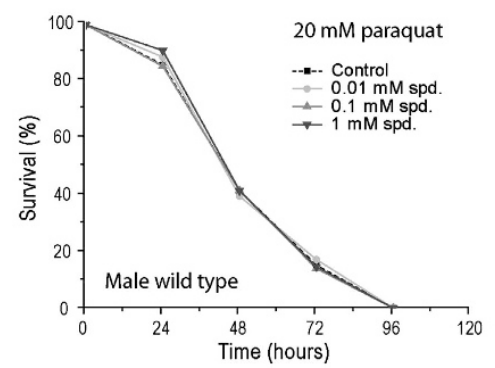

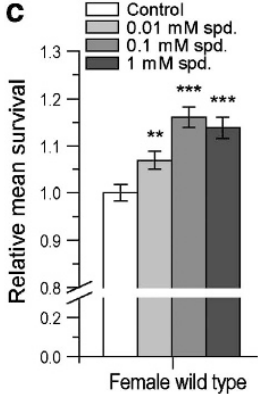
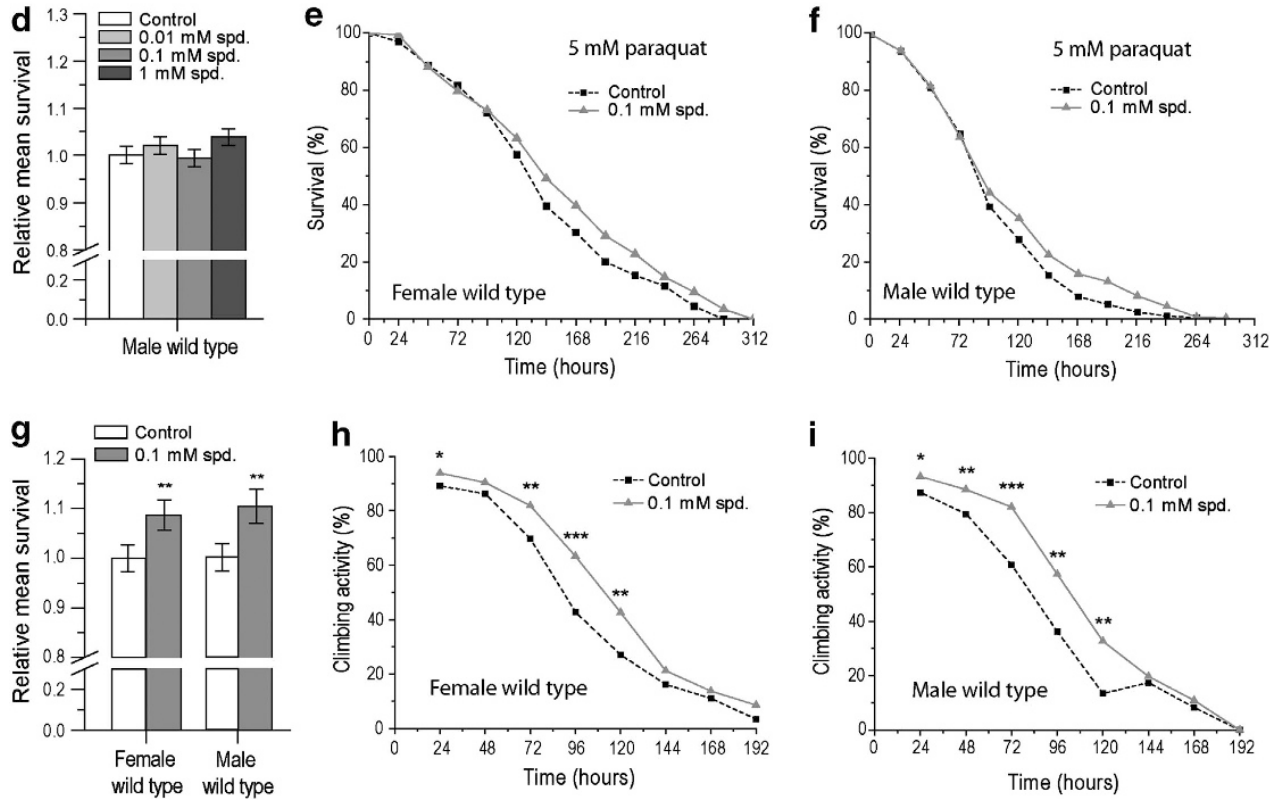

Figure 1 Spermidine-feeding confers paraquat resistance to female Drosophila and improves locomotor activity in female and male flies. (a and $\mathbf{b})$ Survival curve for female (a) and male (b) wild-type flies exposed to $20 \mathrm{mM}$ paraquat and treated with either no spermidine (control), $0.01,0.1$ or $1 \mathrm{mM}$ spermidine (spd.). Results represent the pooled data of five independent replicate experiments. (c and d) Relative mean survival \pm S.E.M. for female (c) and male (d) wild-type flies exposed to $20 \mathrm{mM}$ paraquat and treated with either no spermidine (control), $0.01,0.1$ or $1 \mathrm{mM}$ spd. Mean survival was normalized to the untreated control. Results represent the pooled data of five independent replicate experiments $\left({ }^{* *} P<0.01,{ }^{* \star *} P<0.001\right)$. (e and f) Survival curve for female (e) and male (f) wild-type flies exposed to $5 \mathrm{mM}$ paraquat and treated with either no spd. (control) or $0.1 \mathrm{mM}$ spd. Results represent the pooled data of three independent replicate experiments. (g) Relative mean survival \pm S.E.M. for female and male wild-type flies exposed to $5 \mathrm{mM}$ paraquat and treated with either no spd. (control) or $0.1 \mathrm{mM}$ spd. Mean survival was normalized to the untreated control. Results represent the pooled data of three independent replicate experiments $\left.{ }^{* *} P<0.01\right)$. (h and i) Climbing activity curve for female $(\mathrm{h})$ and male (i) wild-type flies exposed to $5 \mathrm{mM}$ paraquat and treated with or without $0.1 \mathrm{mM}$ spd. Results show the percentage of flies able to climb $8 \mathrm{~cm}$ of the vial in $10 \mathrm{~s}$ and represent the pooled data of three independent replicate experiments $\left({ }^{\star} P<0.05 ;{ }^{* \star} P<0.01 ;{ }^{* *} P<0.001\right)$

the control (Figure 2c, Supplementary Figure S2A). Similarly, paraquat-induced locomotor impairment could not be rescued neither in female nor male atg7-deficient animals (Figures $2 \mathrm{~h}$ and i, Supplementary Figures S2E-J) as opposed to the results obtained in wild-type flies (compare with Figures $1 \mathrm{~h}$ and i). Thus, autophagy is essential for Spdmediated resistance to paraquat-induced toxicity as well as for paraquat-induced loss of locomotor performance.

Spd confers resistance to mild $\mathrm{H}_{2} \mathrm{O}_{2}$ exposure but not to more severe exposure or to starvation. Given that paraquat specifically induces the generation of superoxide anion radicals, we asked whether the observed rescuing effect of Spd was the result of a protection against paraquatspecific or rather general oxidative stress. To test this, we examined survival of wild-type male and female flies in a different oxidative stress situation, namely upon exposure to $\mathrm{H}_{2} \mathrm{O}_{2}$. As observed with $5 \mathrm{mM}$ paraquat, the decrease in survival resulting from challenge to $1 \% \mathrm{H}_{2} \mathrm{O}_{2}$ could be partly rescued by Spd supplementation in both male and female flies (Figures $3 a, b$ and e). However, in contrast to paraquat exposure, Spd treatment also increased survival to $1 \% \mathrm{H}_{2} \mathrm{O}_{2}$ in autophagy-deficient atg $7^{-1-}$ flies (Figures $3 c$, $d$ and $\mathrm{f}$ ). In turn, when $\mathrm{H}_{2} \mathrm{O}_{2}$ was applied at a higher concentration (2\%), Spd supplementation could not revert the adverse effects on survival (Supplementary Figures S3A-D). Similar results were obtained in the atg7-deletion background (Supplementary Figures S3E-H). Spd at the highest concentration even seemed to decrease survival in $\operatorname{atg} 7^{-1-}$ males (Supplementary Figure $\mathrm{S} 3 \mathrm{H}$ ). 

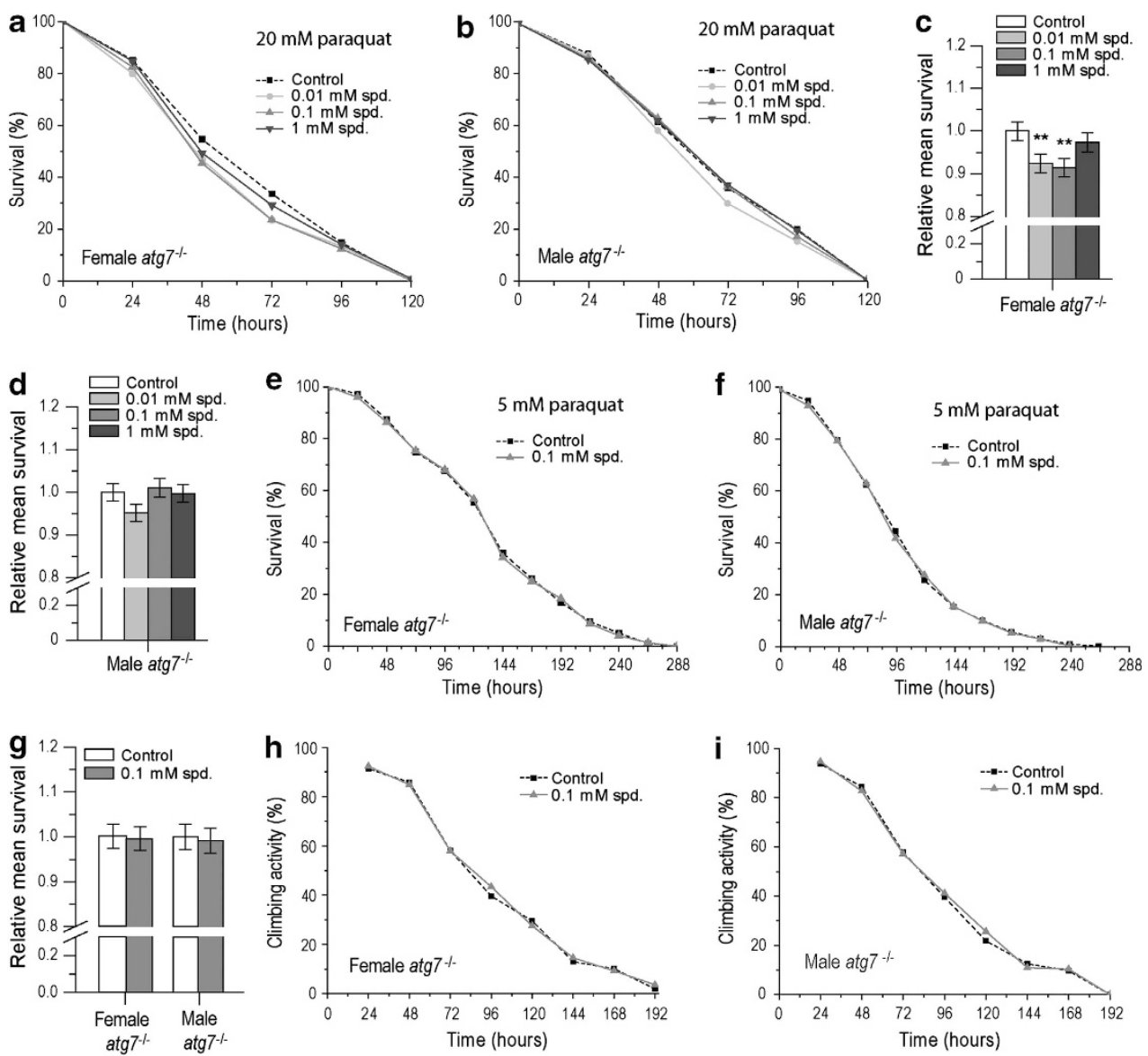

Figure 2 Spermidine treatment does not promote either paraquat resistance or locomotor activity in autophagy-deficient Drosophila. (a and b) Survival curve for female (a) and male (b) atg $7^{-1-}$ flies exposed to $20 \mathrm{mM}$ paraquat and treated with either no spermidine (control), $0.01,0.1$ or $1 \mathrm{mM}$ spermidine (spd.). Results represent the pooled data of five independent replicate experiments. (c and d) Relative mean survival \pm S.E.M. for female (c) and male (d) $\operatorname{atg} 7^{-1-}$ flies exposed to $20 \mathrm{mM}$ paraquat and treated with either no spermidine (control), $0.01,0.1$ or $1 \mathrm{mM}$ spd. Mean survival was normalized to the untreated control. Results represent the pooled data of five independent replicate experiments $\left({ }^{*} P<0.01\right)$. (e and f) Survival curve for female (e) and male (f) atg $7^{-1-}$ flies exposed to $5 \mathrm{mM}$ paraquat and treated with either no spermidine (control) or $0.1 \mathrm{mM}$ spd. Results represent the pooled data of three independent replicate experiments. (g) Relative mean survival \pm S.E.M. for female and male atg $7^{-1-}$ flies exposed to $5 \mathrm{mM}$ paraquat and treated with either no spermidine (control) or $0.1 \mathrm{mM}$ spd. Mean survival was normalized to the untreated control. Results represent the pooled data of three independent replicate experiments. (h and i) Climbing activity curve for female (h) and male (i) atg $7^{-1}$ flies exposed to $5 \mathrm{mM}$ paraquat and treated with or without $0.1 \mathrm{mM}$ spd. Results show the percentage of flies able to climb $8 \mathrm{~cm}$ of the vial in $10 \mathrm{~s}$ and represent the pooled data of three independent replicate experiments

To evaluate if Spd might also exhibit a rescuing effect upon exposure to adverse conditions requiring different mechanisms than oxidative stress, we starved flies by keeping them in vials containing only water and agar with or without Spd and measured survival. Starvation stress resulted in a severe loss of viability over time reaching complete death of the population after approximately 160 and $120 \mathrm{~h}$ in female and male flies, respectively (Supplementary Figures S4A, B). These survival rates remained unchanged also when flies were additionally fed Spd (Supplementary Figures S4A-F). Altogether, these data suggest that Spd specifically reduces oxidative stress as generated through paraquat or mild doses of $\mathrm{H}_{2} \mathrm{O}_{2}$ but does not protect against exposure to severe $\mathrm{H}_{2} \mathrm{O}_{2}$ or starvation stress.

\section{Discussion}

We have previously shown that the polyamine Spd increases life span in various model organisms, as well as in human immune cells. ${ }^{11,19-21}$ Life span extension has often been linked to a concomitant increase in stress resistance. For instance, the first longevity mutant identified, the age-1 mutant in Caenorhabditis elegans, also displayed heat resistance. ${ }^{22}$ More recently, flies mutants in Loco, a Drosophila regulator of G-protein signaling protein, were shown to exhibit a longer life span accompanied by a higher resistance to starvation, heat and paraquat. ${ }^{23}$ Such coupling of longevity and stress resistance is not only observed when life span is increased by mutations but also when achieved by non-genetic interventions. For instance, dietary restriction - a well-known longevity inductor - has been demonstrated to increase stress resistance ${ }^{24}$ and decrease oxidative damage, ${ }^{25}$ although some negative effects of dietary restriction on stress resistance have been reported too. ${ }^{26}$ Similarly, we could show that life span-extending Spd administration increases resistance to heat and $\mathrm{H}_{2} \mathrm{O}_{2}$ in yeast, and decreases agerelated oxidative stress in mice. ${ }^{11}$ Indeed, extensive evidence - especially in plants - supports the concept that polyamines 

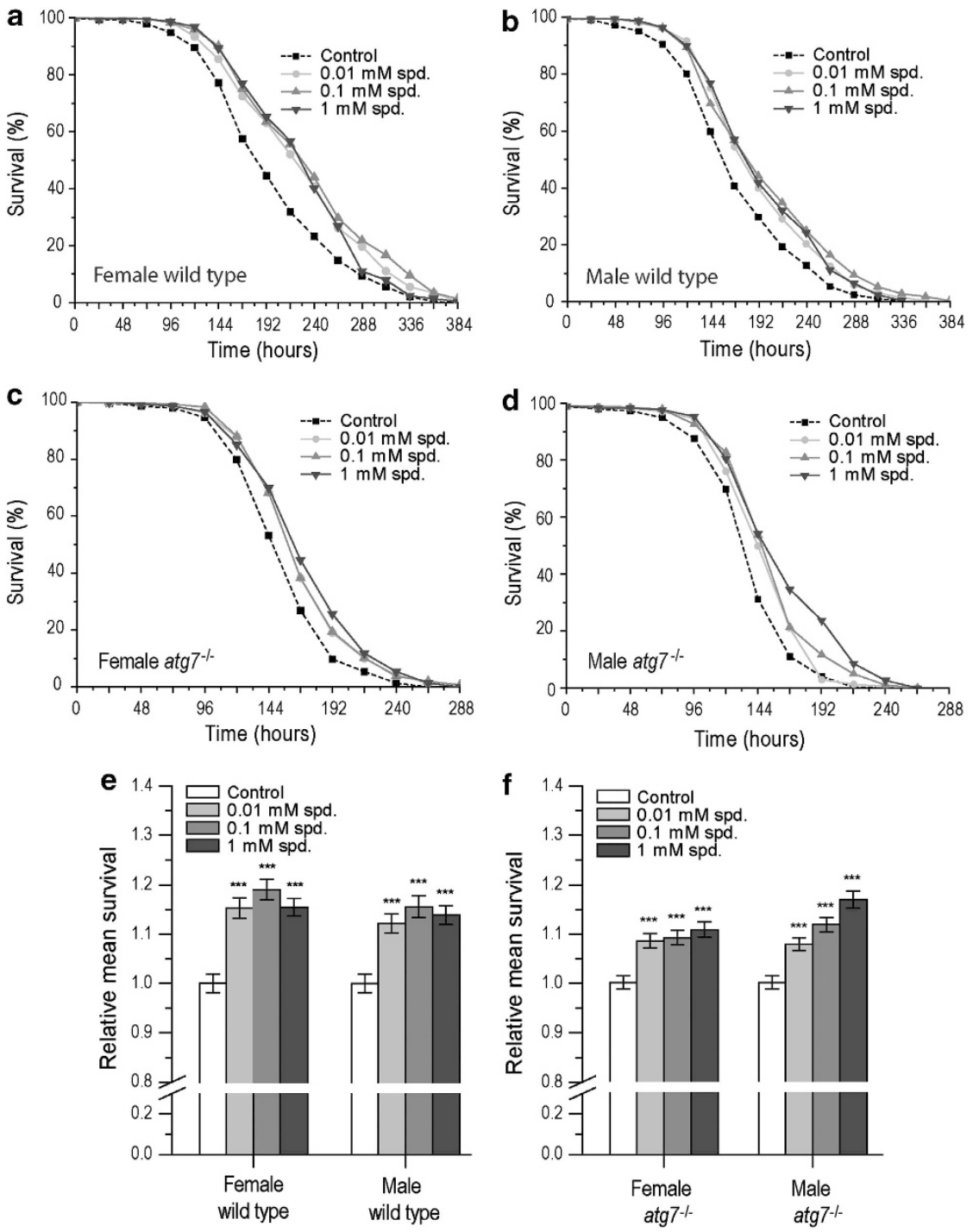

Figure 3 Spermidine treatment promotes resistance to $1 \% \mathrm{H}_{2} \mathrm{O}_{2}$ both in normal and in autophagy-deficient Drosophila. (a and $\left.\mathbf{b}\right)$ Survival curve for female (a) and male (b) wild-type flies exposed to $1 \% \mathrm{H}_{2} \mathrm{O}_{2}$ and treated with either no spermidine (control), $0.01,0.1$ or $1 \mathrm{mM}$ spermidine (spd.). Results represent the pooled data of three independent replicate experiments. (c and d) Survival curve for female (c) and male (d) atg $7^{-1-}$ flies exposed to $1 \% \mathrm{H}_{2} \mathrm{O}_{2}$ and treated with either no spd. (control), $0.01,0.1$ or $1 \mathrm{mM}$ spd. Results represent the pooled data of three independent replicate experiments. (e) Relative mean survival \pm S.E.M. for female and male wild-type flies exposed to $1 \% \mathrm{H}_{2} \mathrm{O}_{2}$ and treated with either no spd. (control), $0.01,0.1$ or $1 \mathrm{mM}$ spd. Mean survival was normalized to the untreated control. Results represent the pooled data of three independent replicate experiments ${ }^{* * *} P<0.001$ ). (f) Relative mean survival \pm S.E.M. for female and male atg ${ }^{-1}$ flies exposed to $1 \% \mathrm{H}_{2} \mathrm{O}_{2}$ and treated with either no spd. (control), $0.01,0.1$ or $1 \mathrm{mM}$ spd. Mean survival was normalized to the untreated control. Results represent the pooled data of three independent replicate experiments $\left.{ }^{* \star \star} P<0.001\right)$

improve stress resistance. ${ }^{21}$ In the present study, we show that Spd confers resistance to paraquat and mild doses of $\mathrm{H}_{2} \mathrm{O}_{2}$ in $D$. melanogaster. Although Spd increased survival of both female and male flies upon exposure to $1 \% \mathrm{H}_{2} \mathrm{O}_{2}$ and $5 \mathrm{mM}$ paraquat, it did so only in females when challenged to a high paraquat concentration $(20 \mathrm{mM})$. In fact, it is generally observed that female flies are more resistant to stress than male flies, most likely because females are bigger and can withstand the stress to which they are exposed longer before dying. Thus, $20 \mathrm{mM}$ paraquat (in contrast to $5 \mathrm{mM}$ ) might be too high of a concentration to withstand for the males but not for the females, allowing only the latter to take advantage of Spd supplementation. Of note, the big difference in size probably explains why we observed the biggest survival difference between sexes on starvation resistance as compared with the other tested stresses: starvation resistance relies entirely on the available bodily reserves of the organism, which in females are larger than in males.

Paraquat is a superoxide generator and thus its toxicity seems to be related at least in part to oxidative damage. This idea is supported by the fact that pure polyphenols, known for their antioxidant properties, increase survival and locomotion in flies exposed to paraquat. ${ }^{27}$ Resveratrol on the other hand, another known antioxidant with life span-extending effects, does not rescue paraquat-induced locomotor impairment in 
flies and even decreases exploratory locomotion in flies not exposed to paraquat. $^{17}$ Thus, establishing the specific mechanism(s) underlying the beneficial effects of Spd on both locomotion and survival upon toxic exposure to paraquat is crucial.

According to our results, it may involve autophagy, an intracellular self-digestion process that we have previously shown to determine Spd-mediated life span extension in yeast, worms and flies. ${ }^{11}$ The present study reveals that upon paraquat exposure the survival and the locomotor activity of autophagy-deficient mutant flies cannot be improved by Spd feeding. Autophagy is thus an essential component of Spdmediated resistance to paraquat. This aligns with reports showing that paraquat exposure induces autophagy as a protective response in models as diverse as the plant Arabidopsis ${ }^{28}$ or human neuroblastoma cells ${ }^{29}$ and seems to be protective. In neuroblastoma cells, inhibition of autophagy accelerates apoptotic cell death, whereas in Arabidopsis, the proteins oxidized by paraquat are degraded by autophagy. It thus appears that autophagy contributes to the faster or more efficient degradation of damaged molecules arising from paraquat exposure conferring longer protection to this stress. It is tempting to speculate that a similar mechanism might underlie Spd-mediated protection against paraquat in flies, which will need to be tested in future studies.

However, autophagy does not seem to be the only mechanism by which Spd is able to confer protection in a given oxidative stress scenario. Interestingly, our data show that while Spd supplementation also increases resistance to a different oxidative stress than paraquat, namely $1 \% \mathrm{H}_{2} \mathrm{O}_{2}$, in this scenario Spd still increases survival in $\operatorname{atg} 7^{-1-}$ flies. Thus, under $\mathrm{H}_{2} \mathrm{O}_{2}$ conditions autophagy does not seem to be involved in the rescuing mechanism. Interestingly, Spd seems to exhibit both autophagy-dependent but also -independent protective effects under different conditions in yeast as well (early and late chronological aging). ${ }^{10,11}$ The discrepancy in the effect of Spd on resistance to paraquat and $\mathrm{H}_{2} \mathrm{O}_{2}$ in flies may be due to the fact that oxidative stress as induced by paraquat, which directly generates superoxide anion radicals, may work by a different mechanism than that triggered by $\mathrm{H}_{2} \mathrm{O}_{2}$. Girardot et al. ${ }^{30}$ reported that almost 10 times as many genes were up or downregulated in Drosophila during paraquat compared with $\mathrm{H}_{2} \mathrm{O}_{2}$ exposure, although both treatments induced the same mortality. Furthermore, some sets of genes were specific to one treatment, supporting the fact that toxicity mechanisms are different for paraquat and $\mathrm{H}_{2} \mathrm{O}_{2}$. For instance, $73 \%$ of the genes encoding the $26 \mathrm{~S}$ proteasome subunits were induced by paraquat but not by $\mathrm{H}_{2} \mathrm{O}_{2}$ treatment. In contrast, ubiquitin protein ligases were under-represented in genes affected by paraquat. The authors hypothesized that Drosophila can implement two types of response to oxidative stress: one relying on posttranscriptional mechanisms as induced by $\mathrm{H}_{2} \mathrm{O}_{2}$ and the other one supported by a coordinated increase of proteasome genes as induced by paraquat. Of note, Spd failed to rescue toxicity resulting from more severe $\mathrm{H}_{2} \mathrm{O}_{2}$ exposure (2\%). This may be due to the fact that the cellular damage inflicted by very high $\mathrm{H}_{2} \mathrm{O}_{2}$ concentrations differs from that resulting from more moderate toxic levels.
It should be noted that in this study autophagy dependency was assayed using $\operatorname{atg} 7^{-1-}$ flies, which are deficient in the essential autophagic regulator ATG7. It has been shown that $\operatorname{atg} 7^{-1-}$ flies are generally shorter-lived, less resistant to diverse stresses (including $30 \mathrm{mM}$ paraquat, $1 \% \mathrm{H}_{2} \mathrm{O}_{2}$ - both mixed in food - and starvation), display faster decline of climbing activity and enhanced neurodegeneration. ${ }^{31}$ Likewise, we have previously reported that $\operatorname{atg} 7^{-1-}$ flies are shorter-lived. ${ }^{11}$ Our herein presented results furthermore show that lack of autophagy decreases overall survival and climbing activity upon $5 \mathrm{mM}$ paraquat exposure (albeit not always significantly) and survival to 1 and $2 \% \mathrm{H}_{2} \mathrm{O}_{2}$. In contrast, some of our results also indicate that lack of autophagy may not always be detrimental. For instance, overall survival to $20 \mathrm{mM}$ paraquat exposure is not affected in $\operatorname{atg} 7^{-1-}$ animals. In fact, lack of autophagy has been already reported to not always lead to dramatic effects; for example, atg $7^{-1-}$ flies do not show any developmental defect and are fully viable. ${ }^{31}$ It may also be that in our study the stress under which ATG7 deletion did not influence overall survival $(20 \mathrm{mM}$ paraquat) was comparatively strong so that the lack of autophagy could not worsen an already low survival.

Finally, we report that the rescuing effect of Spd as observed towards paraquat and $1 \% \mathrm{H}_{2} \mathrm{O}_{2}$ toxicity is absent upon challenge to a further stressor, starvation. The varying degrees of correlations reported between oxidative stress and starvation resistance in long-lived organisms suggest that the machinery involved in executing a response to these two stress factors may or may not overlap depending on the conditions. For example, although Loco mutant female flies were more resistant to both paraquat and starvation, ${ }^{23} \mathrm{Gr} 63 \mathrm{a}$ (a $\mathrm{CO}_{2}$ sensor) female mutant flies were more resistant to paraquat but not to starvation. ${ }^{32}$ Resistance to starvation is mainly controlled by the amount of reserves (lipids and glycogen) of the flies. Thus, metabolic changes induced by Spd supplementation may prevent beneficial effects of Spd on survival to this stress. Although it is yet unknown whether Spd does alter metabolism, some evidence hints towards it. For instance, mice fed a high polyamine diet have been reported to be hyperphagic. ${ }^{13}$ Current work studying the effect of Spd on metabolism in Drosophila will help to clarify a putative role of Spd in metabolism modulation.

To sum up, this study adds to the mounting evidence delineating the beneficial effects of Spd under specific adverse conditions. We show for the first time that Spd confers autophagy-dependent resistance to the neurotoxic agent paraquat by improving survival and locomotor activity in Drosophila. Our work suggests that Spd might counteract neuronal damage caused by particular types of oxidative stress. Future work, however, will have to test this speculation and address the mechanism(s) underlying the herein presented effects. On the other hand, we show that Spd is able to protect against a different type of oxidative stress $\left(\mathrm{H}_{2} \mathrm{O}_{2}\right)$ but in an autophagy-independent manner. Thus, Spd is able to promote survival with or without the involvement of autophagy, depending on the specific oxidative stress scenario.

\section{Materials and Methods}

Flies and reagents. Flies from an isogenized $w^{1118}$ strain were used in all the experiments. The lines for the generation of $A \operatorname{tg} T^{/-}$flies were kindly provided 
by T Neufeld (University of Minnesota, Minneapolis, MN, USA). ${ }^{31}$ The homozygote mutants, $\operatorname{Atg} 7^{d 14} / A \operatorname{tg} 7^{d 77}$, are homozygous mutants for $\operatorname{Atg} 7$. Flies were kept in $25^{\circ} \mathrm{C}, 70 \%$ humidity, $12 \mathrm{~h}$ light $/ 12 \mathrm{~h}$ dark incubator, on an agarcornmeal-sugar-yeast standard diet. Spermidine (Sigma, St. Louis, MO, USA Ref S4139) was prepared as a 1-M stock solution in sterile distilled water, aliquoted in single-use portions and stored at $-20^{\circ} \mathrm{C}$. New stock solution was prepared once a month. Paraquat (Sigma, Ref 856177) was prepared as a 1-M stock solution in sterile distilled water and stored at $4^{\circ} \mathrm{C} . \mathrm{H}_{2} \mathrm{O}_{2}$ (Sigma, Ref $\mathrm{H} 3410$ ) was purchased as a $30 \%$ solution and kept at $4{ }^{\circ} \mathrm{C}$. It was diluted as specified just before use.

Paraquat resistance. Around 20 5-day-old flies were put in each vial containing filter papers (Sigma, Ref Z274852) soaked with $1.5 \mathrm{ml}$ of a solution consisting of $5 \%$ glucose, $20 \mathrm{mM}$ paraquat and either $0,0.01,0.1$ or $1 \mathrm{mM} \mathrm{Spd}$, with an average of 87 flies in each group. Both $\mathrm{w}^{1118}$ and atg $7^{-1-}$ flies were studied, yielding a total of 3622 and 3427 observed flies, respectively. Paraquat resistance was measured in both males and females, which were kept in separate vials. Solutions were renewed every other day until the last fly died. Vials were checked for dead flies every $24 \mathrm{~h}$. Comparison of survivorship for pooled data of all replicates was performed using log rank or Wilcoxon survival tests and corrected for multiple comparisons against the control group. Each genotype and sex was analyzed separately.

Locomotor activity. Ten 5-day-old flies were put in a vial containing filter papers soaked with $1.5 \mathrm{ml}$ of a solution consisting of $5 \%$ glucose, $5 \mathrm{mM}$ paraquat and with or without $0.1 \mathrm{mM}$ Spd with an average of 99 flies in each group. Both $\mathrm{w}^{1118}$ and $\operatorname{atg} 7^{-1-}$ flies were studied, yielding a total of 1179 and 1188 observed flies, respectively. Locomotor activity was measured in both males and females, which were kept in separate vials. Vials were checked for dead flies every $24 \mathrm{~h}$ until the last fly died and filters renewed every other day. Locomotor activity was measured daily until no fly could perform the task. Flies were moved to the bottom of their vial by mechanical stimulation and the number of flies reaching the top of the vial $(8 \mathrm{~cm})$ in $10 \mathrm{~s}$ was recorded. The difference with Spd on the proportion of flies able to perform the climbing task was tested for each sex, genotype and age (hours after beginning of exposure to paraquat) separately using nonparametric $z$ tests for proportions. Comparison of survivorship of pooled data was performed using log-rank survival tests. Each genotype and sex was analyzed separately.

Hydrogen peroxide resistance. Around 20 5-day-old flies were put in a vial in containing filter papers soaked with $1.5 \mathrm{ml}$ of a solution consisting of $5 \%$ glucose and 1 or $2 \% \mathrm{H}_{2} \mathrm{O}_{2}$ without or with either $0.01,0.1$ or $1 \mathrm{mM} \mathrm{Spd}$, with an average of 97 flies in each group for $1 \% \mathrm{H}_{2} \mathrm{O}_{2}$ and 98 flies in each group for $2 \%$. Both $\mathrm{w}^{1118}$ and $\operatorname{atg} 7^{-1-}$ flies were studied, yielding a total of 2329 and 2353 observed flies, respectively, for $1 \% \mathrm{H}_{2} \mathrm{O}_{2}$, and 2360 and 2356 observed flies, respectively, for $2 \% \mathrm{H}_{2} \mathrm{O}_{2} . \mathrm{H}_{2} \mathrm{O}_{2}$ resistance was measured in both males and females, which were kept in separate vials. Solutions were renewed every other day until the last fly died. Vials were checked for dead flies every $24 \mathrm{~h}$. Comparison of survivorship data of pooled data was performed using log rank or Wilcoxon survival tests. Each genotype and sex was analyzed separately.

Starvation resistance. Around 20 5-day-old flies were put in a vial containing only water and agar without or with either $0.01,0.1$ or $1 \mathrm{mM} \mathrm{Spd}$, with an average of 99 flies in each group. Only $w^{1118}$ flies were studied, yielding a total of 2382 observed flies. Starvation resistance was measured in both males and females, which were kept in separate vials. Vials were renewed every other day until the last fly died. Vials were checked for dead flies every $24 \mathrm{~h}$. Comparison of survivorship of pooled data was performed using log rank or Wilcoxon survival tests. Each sex was analyzed separately.

\section{Conflict of Interest}

The authors declare no conflict of interest.

Acknowledgements. This work was supported by the University of St. Andrews, as well as by the Austrian Science Fund FWF (grants LIPOTOX and
P23490-B12 to FM; P24381-B20 to FM and TE) and the European Commission (Apo-Sys to FM and TE). Furthermore, this work was supported by grants to GK from the Ligue Nationale contre le Cancer (Equipe labellisée), Agence Nationale pour la Recherche (ANR), Cancéropôle lle-de-France, European Commission (ApoSys, ArtForce), Fondation Axa (Chair for longevity research), Fondation pour la Recherche Médicale (FRM), Institut National du Cancer (INCa), et Fondation Bettencourt-Schueller.

1. Vaupel JW. Biodemography of human ageing. Nature 2010; 464: 536-542.

2. Kenyon CJ. The genetics of ageing. Nature 2010; 464: 504-512.

3. Fontana L, Partridge L, Longo VD. Extending healthy life span-from yeast to humans. Science 2010; 328: 321-326.

4. Baur JA, Pearson KJ, Price NL, Jamieson HA, Lerin C, Kalra A et al. Resveratrol improves health and survival of mice on a high-calorie diet. Nature 2006; 444: 337-342.

5. Miller RA, Harrison DE, Astle CM, Baur JA, Boyd AR, de Cabo R et al. Rapamycin, but not resveratrol or simvastatin, extends life span of genetically heterogeneous mice. $J$ Gerontol 2011; 66: 191-201.

6. Harrison DE, Strong R, Sharp ZD, Nelson JF, Astle CM, Flurkey K et al. Rapamycin fed late in life extends lifespan in genetically heterogeneous mice. Nature 2009; 460: 392-395.

7. Bjedov I, Toivonen JM, Kerr F, Slack C, Jacobson J, Foley A et al. Mechanisms of life span extension by rapamycin in the fruit fly Drosophila melanogaster. Cell Metab 2010; 11: $35-46$.

8. Harrison B, Tran TT, Taylor D, Lee SD, Min KJ. Effect of rapamycin on lifespan in Drosophila. Geriatr Gerontol Int 2010; 10: 110-112.

9. Nishimura K, Shiina R, Kashiwagi K, Igarashi K. Decrease in polyamines with aging and their ingestion from food and drink. J Biochem 2006; 139: 81-90.

10. Carmona-Gutiérrez D, Bauer MA, Ring J, Knauer $\mathrm{H}$, Eisenberg $\mathrm{T}$, Büttner $\mathrm{S}$ et al. The propeptide of yeast cathepsin D inhinbits programmed necrosis. Cell Death Dis 2011; 19: e161.

11. Eisenberg T, Knauer H, Schauer A, Fussi H, Büttner S, Carmona-Gutierrez D et al. Induction of autophagy by spermidine promotes longevity. Nat Cell Biol 2009; 11: 1305-1314.

12. Suppola S, Heikkinen S, Parkkinen JJ, Uusi-Oukari M, Korhonen VP, Keinänen T et al. Concurrent overexpression of ornithine decarboxylase and spermidine/spermine $\mathrm{N}^{1}$-acetyltransferase further accelerates the catabolism of hepatic polyamines in transgenic mice. Biochem J 2001; 358: 343-348.

13. Soda K, Dobashi Y, Kano Y, Tsujinaka S, Konishi F. Polyamine-rich food decreases age-associated pathology and mortality in aged mice. Exp Gerontol 2009; 44: 727-732.

14. Kaeberlein M. Spermidine surprise for a long life. Nat Cell Biol 2009; 11: 1277-1278.

15. Gonidakis S, Finkel SE, Longo VD. Lifespan extension and paraquat resistance in a ubiquinone-deficient Escherichia coli mutant depend on transcription factors ArcA and TdcA. Aging 2011; 3: 291-303.

16. Fabrizio P, Pozza F, Pletcher SD, Gendron CM, Longo VD. Regulation of longevity and stress resistance by Sch9 in yeast. Science 2001; 292: 288-290.

17. Bagatini PB, Saur L, Rodrigues MF, Bernardino GC, Paim MF, Coelho GP et al. The role of calcium channel blockers and resveratrol in the prevention of paraquat-induced parkisonism in Drosophila melanogaster. a locomotor analysis. Invert Neurosci 2011; 11: $43-51$.

18. Vellai T. Autophagy genes and ageing. Cell Death Differ 2009; 16: 94-102.

19. Morselli E, Mariño G, Bennetzen MV, Eisenberg T, Megalou E, Schroeder S et al. Spermidine and resveratrol induce autophagy by distinct pathways converging on the acetylproteome. J Cell Biol 2011; 192: 615-629.

20. Madeo F, Eisenberg T, Büttner S, Ruckenstuhl C, Kroemer G. Spermidine: a novel autophagy inducer and longevity elixir. Autophagy 2010; 6: 160-162.

21. Minois N, Carmona-Gutierrez D, Madeo F. Polyamines in aging and disease. Aging 2011; 3: $716-732$.

22. Lithgow GJ, White TM, Melov S, Johnson TE. Thermotolerance and extended life-span conferred by single-gene mutations and induced by thermal stress. Proc Natl Acad Sci USA 1995; 92: 7540-7544.

23. Lin YR, Kim K, Yang Y, Ivessa A, Sadoshima J, Park Y. Regulation of longevity by regulator of G-protein signaling protein, Loco. Aging Cell 2011; 10: 438-447.

24. Lee GD, Wilson MA, Zhu M, Wolkow CA, de Cabo R, Ingram DK et al. Dietary deprivation extends lifespan in Caenorhabditis elegans. Aging Cell 2006; 5: 515-524.

25. Reverter-Branchat G, Cabiscol E, Tamarit J, Ros J. Oxidative damage to specific proteins in replicative and chronological-aged Saccharomyces cerevisiae: common targets and prevention by calorie restriction. J Biol Chem 2004; 279: 31983-31989.

26. Burger JM, Hwangbo DS, Corby-Harris V, Promislow DE. The functional costs and benefits of dietary restriction in Drosophila. Aging Cell 2007; 6: 63-71.

27. Jimenez-Del-Rio M, Guzman-Martinez C, Velez-Pardo C. The effects of polyphenols on survival and locomotor activity in Drosophila melanogaster exposed to iron and paraquat. Neurochem Res 2010; 35: 227-238. 
28. Xiong $Y$, Contento AL, Nguyen $P Q$, Bassham DC. Degradation of oxidized proteins by autophagy during oxidative stress in Arabidopsis. Plant Physiol 2007; 143: 291-299.

29. González-Polo RA, Niso-Santano M, Ortíz-Ortíz MA, Gómez-Martín A, Morán JM

García-Rubio $L$ et al. Inhibition of paraquat-induced autophagy accelerates the apoptotic cell death in Neuroblastoma SH-SY5Y cells. Toxicol Sci 2007; 97: 448-458.

30. Girardot F, Monnier V, Tricoire H. Genome wide analysis of common and specific stress responses in adult Drosophila melanogaster. BMC Genomics 2004; 5: 74.

31. Juhász G, Ėrdi B, Sass M, Neufeld TP. Atg7-dependent autophagy promotes neuronal health, stress tolerance, and longevity but is dispensable for metamorphosis in Drosophila. Genes Dev 2007; 21: 3061-3066.
32. Poon PC, Kuo TH, Linford NJ, Roman G, Pletcher SD. Carbon dioxide sensing modulates lifespan and physiology in Drosophila. PLoS Biol 2010; 8: e1000356.

Cell Death and Disease is an open-access journal SOMERाHWHSRESERVED published by Nature Publishing Group. This work is
licensed under the Creative Commons Attribution-NonCommercialShare Alike 3.0 Unported License. To view a copy of this license, visit http://creativecommons.org/licenses/by-nc-sa/3.0/

Supplementary Information accompanies the paper on Cell Death and Disease website (http://www.nature.com/cddis) 